\title{
The status and performance of Cosmic Ray Air Fluorescence Fresnel lens Telescope (CRAFFT) for the next generation UHECR observatory
}

\author{
Yuichiro Tameda ${ }^{* a}$, Takayuki Tomida $^{b},{\text { Daisuke } \text { Ikeda }^{c}, \text { Katsuya Yamazaki }}^{d}$, Mashu \\ Yamamoto $^{b}$, Hirokazu Iwakura $^{b}$, Yuya Nakamura $^{b}$ and Yuki Kaino ${ }^{a}$ \\ ${ }^{a}$ Department of Engineering Science, Faculty of Engineering, Osaka Electro-Communication \\ University \\ Neyagawa-shi, Osaka, Japan \\ ${ }^{b}$ Academic Assembly School of Science and Technology Institute of Engineering, Shinshu \\ University \\ Nagano, Nagano, Japan \\ ${ }^{c}$ Earthquake Research Institute, University of Tokyo \\ Bunkyo-ku, Tokyo, Japan \\ ${ }^{d}$ Faculty of Engineering, Kanagawa University \\ Yokohama, Kanagawa, Japan \\ E-mail: Eamedadosakac.ac.jp
}

\begin{abstract}
The next generation ultra-high energy cosmic ray (UHECR) observatory should be expanded due to the very small flux. In order to realize a huge UHECR observatory, cost reduction of detectors is one of the useful strategy. Then we are developing a Cosmic Ray Air Fluorescence Fresnel lens Telescope (CRAFFT) which is a very simple structure fluorescence detector. CRAFFT detector consists of mainly a $1.4 \mathrm{~m}^{2}$ Fresnel lens, an UV transmitting filter, an 8 inch photomultiplier tube, an FADC board, and power supply system. We tested CRAFFT detector performance at Telescope Array (TA) site, and succeeded to detect UHECR air showers synchronized with TA Fluorescence Detectors. CRAFFT detector will be deployed as a huge ground array with $\sim 20 \mathrm{~km}$ spacing without the enough infrastructure such as in desert. Therefore, CRAFFT detectors can stand alone. We are developing automatic observation and protecting detector system. Additionally, we are studying our detector performance by detector simulation to estimate trigger efficiency, establish a reconstruction procedure, and optimize our detector configuration. In this presentation, we will report the result of the test operation of automatic observation and protecting detector system for CRAFFT detector, and the detector performance and optimization.
\end{abstract}

36th International Cosmic Ray Conference -ICRC2019-

July 24th - August 1st, 2019

Madison, WI, U.S.A.

${ }^{*}$ Speaker. 


\section{Introduction}

Since the discovery of cosmic rays, they have been observed over a very wide energy range. However, the origin of cosmic rays has not been identified directly yet. Because it interacts with the magnetic field in space and the trajectory is diffused. However, it is expected that cosmic rays with energy above $10^{19} \mathrm{eV}$ are hardly diffused even in the magnetic field in space. Telescope Array (TA) collaboration reported that there is a hotspot region in the arrival direction of cosmic rays with energy above $57 \mathrm{EeV}[\mathrm{W}]$. Then, it is expected that the origin will be clarified by observing such ultra-high energy cosmic rays with higher statistics.

The following cases are necessary to make the arrival direction of cosmic rays correspond to the origin of the source. It is that the primary cosmic rays can propagate straight. In that case, the primary cosmic ray must be a light nucleus like a proton or Helium. So far, the mass composition of cosmic rays has been measured by various methods. The most traditional and reliable mass composition analysis is $X_{\max }$ analysis. $X_{\max }$ is an atmospheric depth where the number of particle in an air shower reaches maximum. The average or fluctuation of $X_{\max }$ is a parameter strongly dependent on mass composition. Because fluorescence detector can measure the longitudinal profile of cosmic ray air shower, fluorescence detector is useful for measuring energy and $X_{\max }$. Although the statistics of fluorescence detector is relatively much lower than a surface detector array due to the low duty cycle. Therefore, in order to determine the mass composition, observation by the fluorescence detectors is effective. TA and HiRes experiments reported that the composition is light such as proton or Helium above $10^{19} \mathrm{eV}[\mathrm{[}][[]]$, but the Auger experiment shows that the composition becomes heavier above $10^{18.5} \mathrm{eV}$ [䧃]. There are also still problems to be solved, such as systematic errors due to hadron interaction models. In order to clarify the sources of ultra-high energy cosmic rays, we have to understand the mass composition and need much more statistics.

In the future, it is inevitable to expand the scale of observatory for ultra-high energy cosmic ray research. Additionally, we also need mass composition sensitive detector such as fluorescence detector until we conclude the mass composition. For this purpose, the concept of low cost fluorescence detector is originally proposed by privitera [ []]. The low cost fluorescence detector is very simple structure consisting of a Fresnel lens, UV transmitting filter and a large photo multiplier tube (PMT). Based on this concept, we are developing a Fresnel lens Fluorescence detector. FAST project is also developing a low cost FD consisting of mirrors and PMTs [回]. As other strategy for ultra-high energy cosmic ray observation, fluorescence detector from space are also under development $[[][[]][Q]$.

\section{CRAFFT}

Cosmic Ray Air Fluorescence Fresnel lens Telescope (CRAFFT) is a fluorescence detector using a Fresnel lens [[0]]. In order to realize the next generation ultra-high energy cosmic ray observatory, it is necessary to make the observation volume much larger with more telescopes. Therefore, cost reduction can not be avoided. On the other hand, in order to measure the mass composition together, a fluorescence detector which can measure $X_{\max }$ is preferable. CRAFFT realizes a low-cost fluorescence detector as follows. The structure is simplified by using a refracting telescope instead of a reflecting telescope, and reducing the components such as the number of 


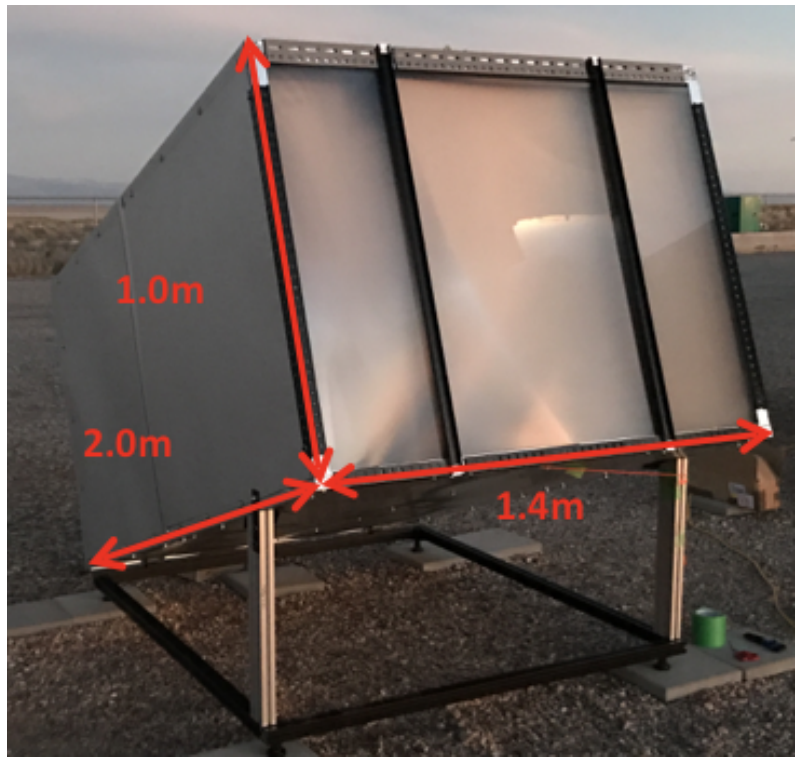

Figure 1: The appearance of CRAFFT detector. The structure is made of anodized T-slotted aluminum extrusions. UV transmitting Fresnel lens is installed at the aperture. The inside of the detector is protected by the steel plate.

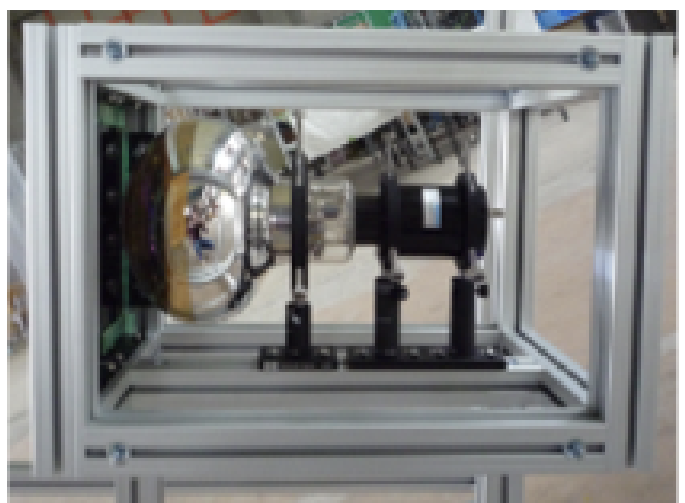

Figure 2: PMT mount installed at the focus of the Fresnel lens. In front of the PMT photocathode, the UV transmitting filter is mounted.

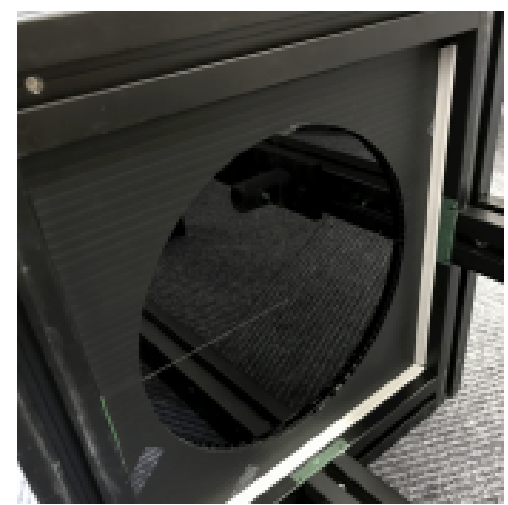

Figure 3: The spacial filter to limit the F.O.V. to reduce the uncertainty of the photon sensitivity at the edge of the PMT.

pixel. Installation is very easy because of its simple structure. Operation cost should be reduced by automatic observation system.

The CRAFFT detector mainly consists of a Fresnel lens, a UV transmission filter, and an 8inch PMT. The detailed components of the CRAFFT detector are listed in the table $\mathbb{W}$. Figure $\square$ shows the appearance of the detector. The structure of the detector is made of black anodized aluminum frame. The surrounding area is covered with a steal plate to protect and shield the inside of the detector. A roll curtain is attached to behind the Fresnel lens and shields the inside in daytime. At the aperture, two beams were attached to prevent the deflection of the Fresnel lens. The elevation of the detector is adjustable. At the focal point of the Fresnel lens, a UV transmission filter as shown in Fig. $\square$ and an 8-inch PMT are installed. The aluminum frame attached to the PMT is also black 
anodized. Additionally, as shown in Fig. [3, a spatial filter is attached to the UV transmission filter to make the field of view narrower than the original design for the test observation. The field of view (F.O.V.) becomes 8 degrees, and originally about 12 degrees without the spacial filter. Because the surface of the PMT is not flat but spherical, it is to reduce the sensitivity ambiguity around the PMT edge. The signal from the PMT is amplified by an amplifier and digitized and stored by a 12 bit and $80 \mathrm{MHz}$ sampling rate FADC board.

Table 1: Component list of the CRAFFT detector

\begin{tabular}{l|l} 
Component & Product \\
\hline \hline Fresnel lens & NTKJ, CF1200-B \\
\hline UV-transmitting filter & Hoya, U330 \\
\hline Photomultiplier tube & Hamamatsu, R5912 \\
\hline HV power supply & CAEN, N1470AR \\
\hline HV divider & (special order) \\
\hline FADC board & TokushuDenshiKairo, Cosmo-Z \\
\hline Low pass filter & Mini Circuit, BLP-15+ \\
\hline Amplifier & LeCroy, MODEL 612AM \\
\hline Structure & YUKI, anodized T-slotted aluminum extrusions \\
\hline
\end{tabular}

\section{Test observation}

In November 2017, test observations were performed at the TA experimental site [प]]. TA is an ultra-high energy cosmic ray observation experiment and is located in the western desert area of Utah, USA. Four CRAFFT detectors were installed beside the TA Fluorescence Detector (FD) station at Black Rock Mesa as shown in Fig. W. The total observation time is 63 hours. To ensure that cosmic ray signals are acquired, test observations are performed while TA FD observation period in order to acquired the trigger timing signal from the trigger electronics of TA FD [12]. The TA experiment recognizes the cosmic ray air shower track every $12.8 \mu \mathrm{s}$. By this test observations, 456,727 events were recorded and we found 10 apparent air shower events after the event selection [[0]]. One of the typical event is given in Fig. [- The black lines in the left panel of Fig. [ represent the field of view of the TA FDs, and the small black circles represent TA FD pixels that recorded cosmic ray air shower signals. The large 4 circles correspond to the field of view of 4 CRAFFT detectors. The right panel of Fig. $\square$ is the waveform of the air shower detected by four CRAFFT detectors. Thus, we have demonstrated the ability to detect ultra-high energy cosmic ray air showers with a very simple fluorescence detector such as CRAFFT.

\section{Development of automation system}

In order to reduce costs, an automatic observation system is essential. To reduce operating costs, what required of an automatic observation system are an electric power supply system, a detector protection system, and an automation data acquisition system with an environment monitor. 


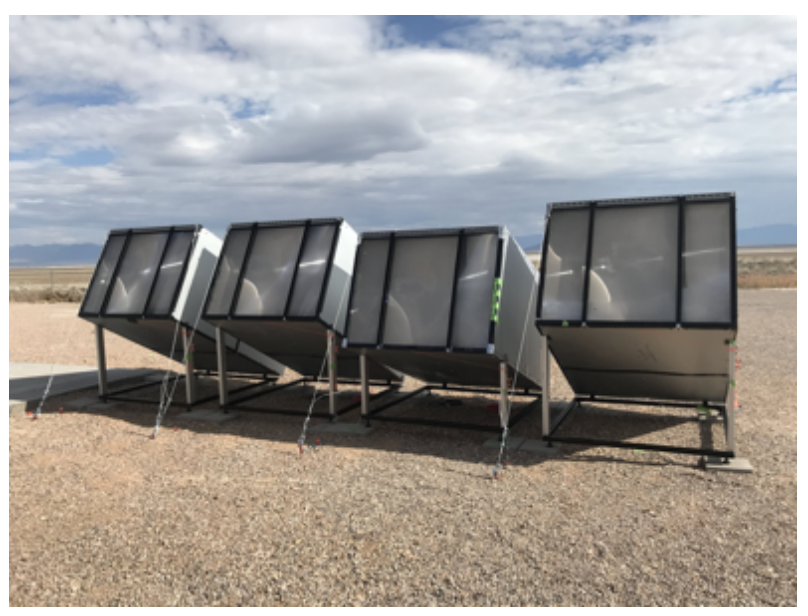

Figure 4: The CRAFFT detectors deployed at the Telescope Array site of Black Rock Mesa, for the test observation.
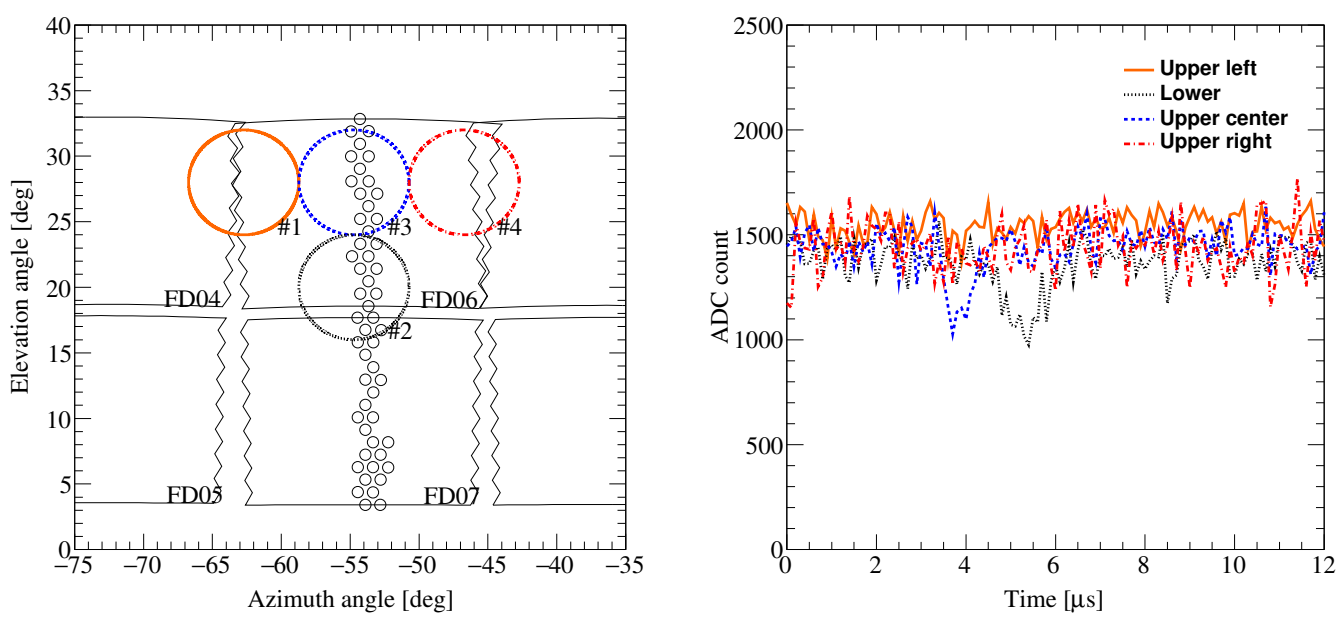

Figure 5: One of the air shower event detected by the CRAFFT detectors. Left: The comparison of the F.O.V. of CRAFFT and TA FD. The bigger circles and solid lines are the edge of the F.O.V. of CRAFFT and TA FD, respectively. The smaller circles are the TA FD PMTs with the signal of the air shower event.

For ultra-high energy cosmic ray observation, it is necessary to install a detector over a very wide area. The installation site will be a place without power such as the desert as the TA experiment is. Thus, the power supply system is needed. The power supply system of the CRAFFT detector consists of a solar panel, a charge controller, and a battery. The solar panels are $300 \mathrm{~W}$ and $24 \mathrm{~V}$. There are three batteries of $12 \mathrm{~V}$ and $100 \mathrm{Ah}$. This system is estimated to allow observation even if it can not be charged for three days due to bad weather.

Data acquisition systems are divided into host electronics and local electronics. The host electronics manages four telescopes using a Raspberry Pi. The power supply is managed by a host electronic relay circuit. Linux is installed on the FADC board used for data acquisition, and can be controlled by the host electronics via a network. Astronomical twilight is judged from the time, and the process of data acquisition is executed automatically. The host electronics is connected to 
the outside through the network. The local electronics applies a high voltage power supply to the PMT and amplifies the signal from the PMT with an amplifier, and connected to the FADC board.

The tests of the power supply system and the data acquisition system were performed in October-December 2018 at TA site. With this system, we succeeded to operate automatically from the initialization of the electronics to data acquisition. Air shower events have not been detected due to the short observation time. However, we found an air shower like events caused by the laser emitted from the Central Laser Facility used for atmospheric calibration of the TA experiment [[13].

The CRAFFT detector is intended to be installed directly on the ground, not inside the building. Therefore, in order to protect the inside of the detector, it is necessary to block incident light from the lens during the daytime. In the 2017 test observation, a roll curtain was installed behind the lens. However, when we measured the change of the transmittance of the lens sample exposed at the CRAFFT detector, it was found that the transmittance decreased by 10 to $15 \%$ per year. Therefore, since it is necessary to protect the lens, we shield light from the outside of the lens. We are currently testing low-power electric shutters that can be remotely controlled shown in Fig. 6 .

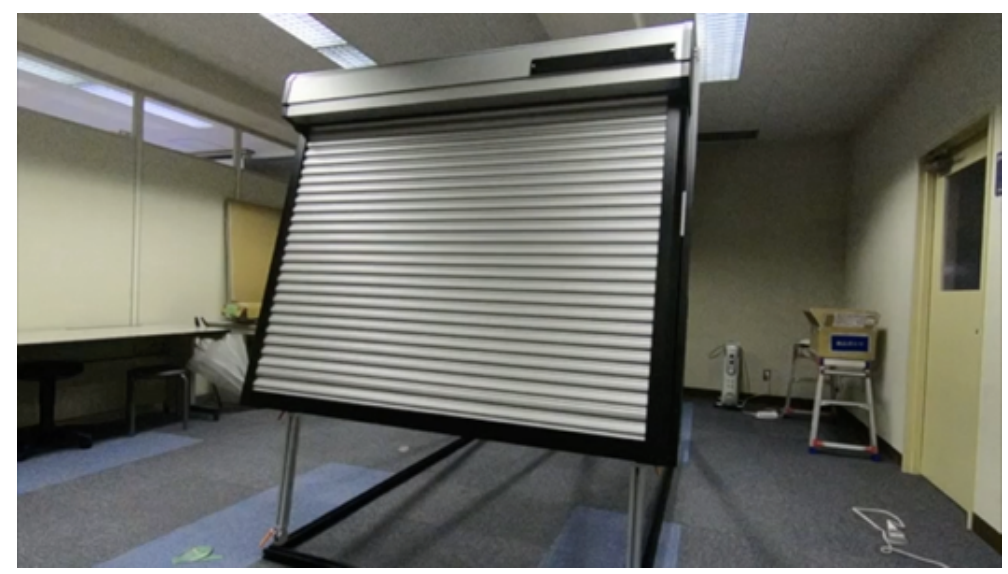

Figure 6: The shutter to protect the Fresnel lens and the inside of the CRAFFT detector. This shutter can be controlled remotely.

\section{Detector performance}

To establish detector configuration optimization, detection efficiency, and analysis method we have developed a detector simulation. Ray-trace simulation was developed based on ROBAST [14]. The spot of the CRAFFT detector has a unique structure as shown in Fig. $\square$. The simulation could reproduce the shape of the spot. The simulated spot at the focal position is shown in Fig. 8 . The spot size including the $95 \%$ light intensity is $44 \mathrm{~mm}$ in the wavelength range of $280-400 \mathrm{~nm}$ containing the wavelength of atmospheric fluorescence light.

The waveform of cosmic ray air shower detected by CRAFFT is simulated. In order to reduce the calculation time in simulation, a database of intensity map of incident light for each incident angle on the PMT surface obtained by ray tracing shown in Fig. $Q$ is used. The nightsky background used in the simulation is acquired by the test observation at Utah. Cosmic ray air shower events observed by CRAFFT are simulated using geometry obtained from TA FD. Figure $\mathbf{U}$ is a 

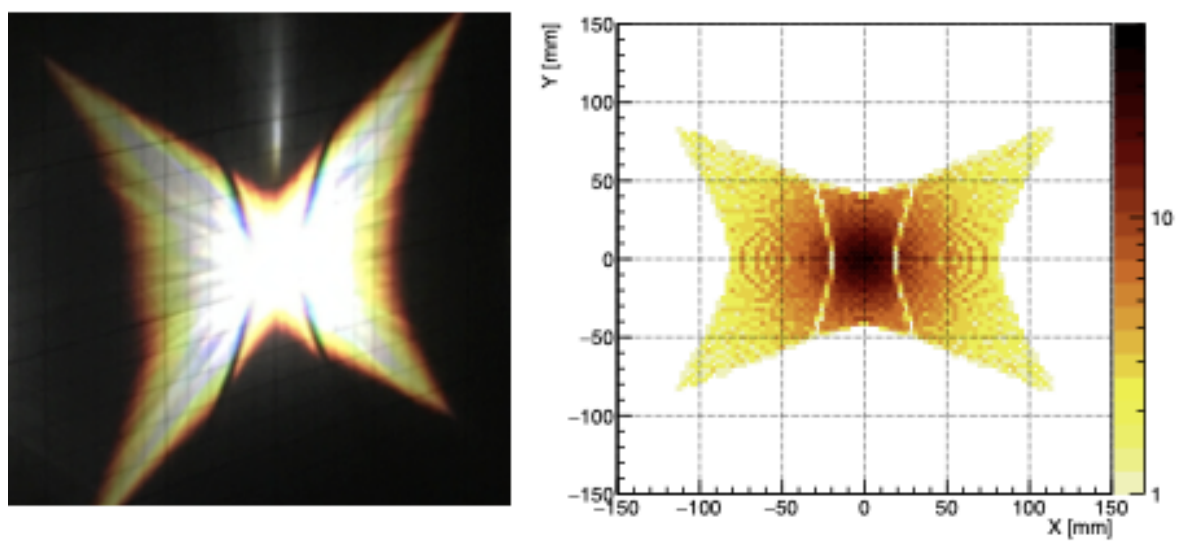

Figure 7: Left: The unique shape of the spot at the screen. Right: The simulated spot shape reproducing the actual spot shape.

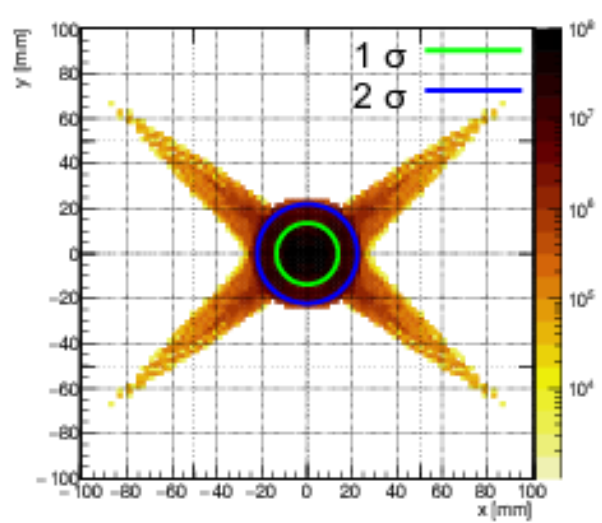

Figure 8: The shape of spot at the focus of the Fresnel lens of CRAFFT detector. The $95 \%$ spot size is $44 \mathrm{~mm}$ shown as blue circle.

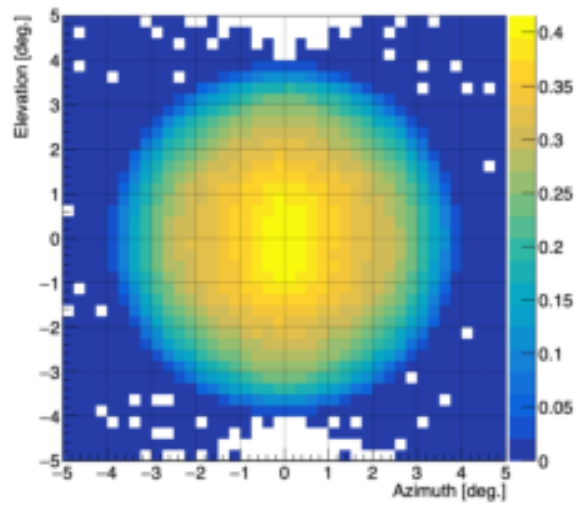

Figure 9: The intensity map of the incident light for each direction at the surface of the PMT at the focus.

comparison of a waveform obtained by the test observation and simulation. The simulated waveform is well reproduced compared to the actual waveform. The detection efficiency was estimated by the simulation with a following conditions. Energies of the primary cosmic ray are $10^{19} \mathrm{eV}$ and $10^{20} \mathrm{eV}$. The zenith angle is less than $65^{\circ}$, and azimuth angle is uniformly. The shower core position is within $30 \mathrm{~km}$ from the CRAFFT detector. We use CORSIKA for air shower simulation [I5]. Primary particles are assumed to be protons, and hadronic interaction model is QGSJETII-04. There are four detectors, and the centers of elevation angles are $4^{\circ}, 12^{\circ}, 20^{\circ}, 28^{\circ}$, and all azimuth angles are the same. Figure $\mathbb{W}$ is a scatter plot of the core position of simulated events with at least a photon incident to the detector and the color represents the $\mathrm{S} / \mathrm{N}$ ratio. The CRAFFT detector is located at the origin in the plot. From this simulation, the distances between the core position and the detector at which the $\mathrm{S} / \mathrm{N}$ ratio becomes about 10 sigma are $15 \mathrm{~km}$ and $30 \mathrm{~km}$, for 
$10^{19} \mathrm{eV}$ and $10^{20} \mathrm{eV}$, respectively.
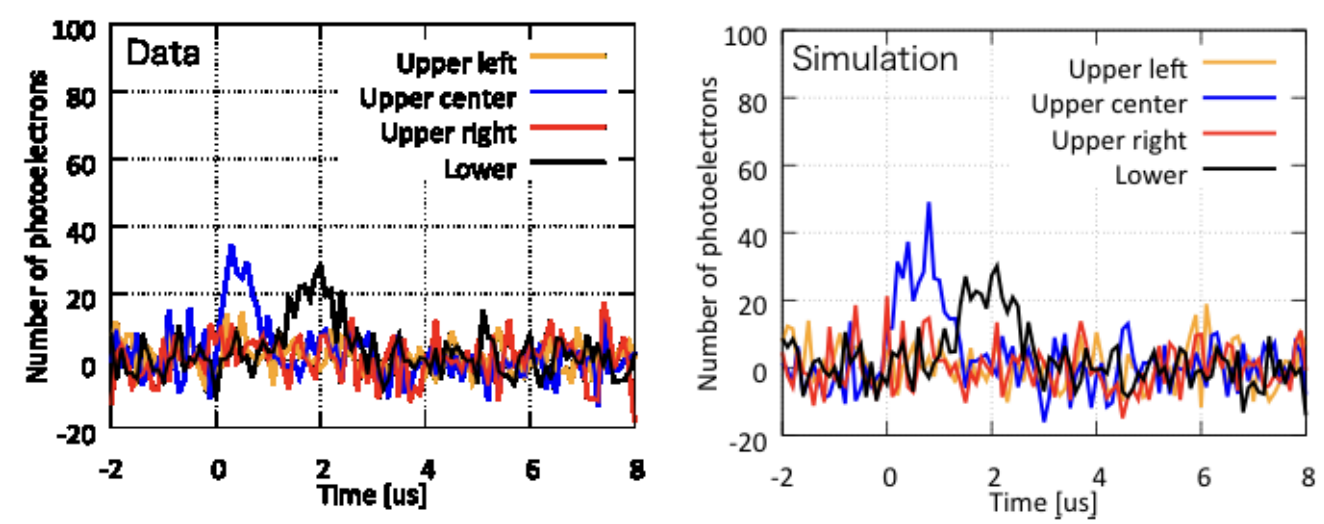

Figure 10: The comparison of the data and simulated waveform of the air shower event. The left is the data, and the right is the simulated waveform.

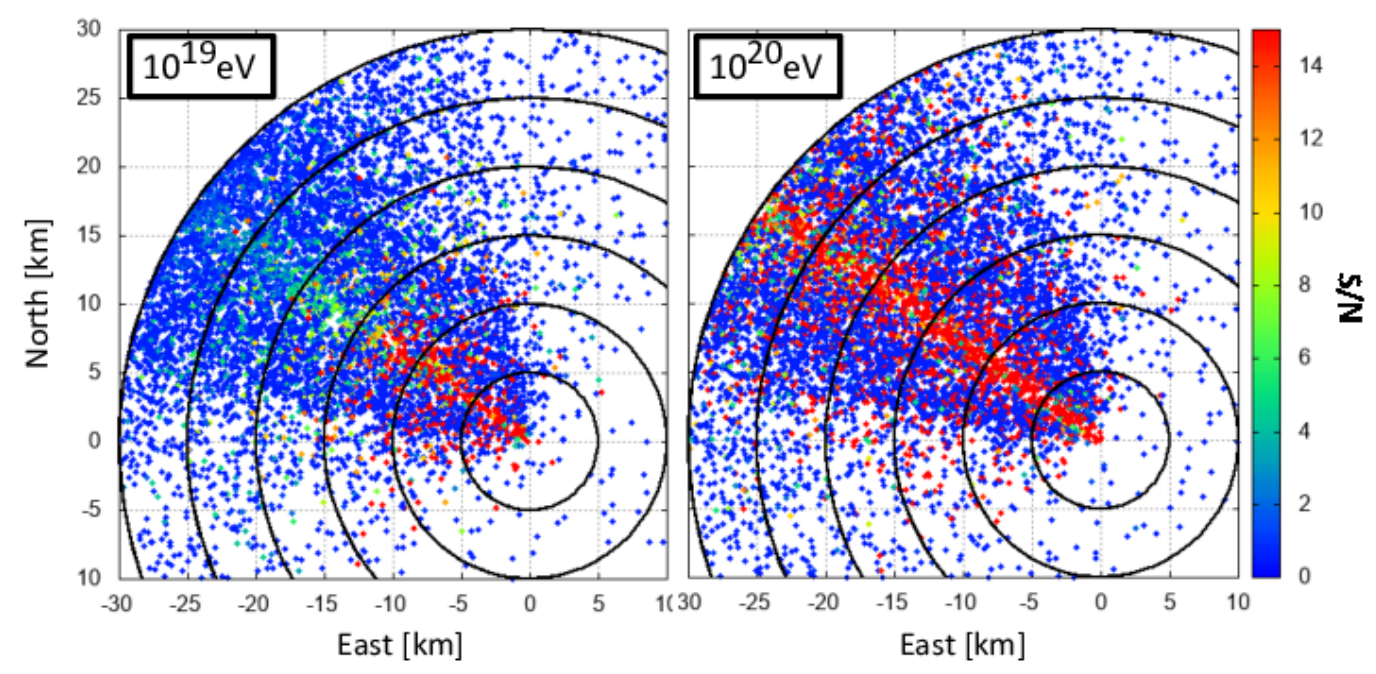

Figure 11: The scatter plot of the simulated air shower core positions. The color variation is corresponding the $\mathrm{S} / \mathrm{N}$ ratio.

\section{Conclusion}

We have developed a simple structure and low cost fluorescence detector for the next generation ultra-high energy cosmic ray observatory named as Cosmic Ray Air Fluorescence Fresnel lens Telescope (CRAFFT). We installed 4 CRAFFT detectors at TA FD site, and we tested the performance in November 2017 and automation data acquisition system in October-December 2018. We succeeded to detect 10 apparent ultra-high energy cosmic ray air showers. The automation data acquisition system also worked well. We demonstrated that CRAFFT detector is one of the candidate detector for the next generation ultra-high energy cosmic ray observatory. 


\section{Acknowledgment}

This work was supported by the JSPS KAKENHI Grant Numbers 25610051 and JP16K17710. This work was partially carried out by the joint research program of the Institute for Cosmic Ray Research (ICRR), The University of Tokyo. This study was also supported by the Earthquake Research Institute The University of Tokyo Joint Usage/Research Program. The Telescope Array Collaboration supported CRAFFT as an associated experiment and allowed us to use TA equipments and FD event displays. We wish to thank the staffs at the University of Utah, especially Prof. J.N. Matthews.

\section{References}

[1] R.U. Abbasi et al., The Astrophysical Journal Letters 790, 2 (2014).

[2] R.U. Abbasi et al., Astropart. Phys. 64, 49-62 (2015).

[3] R.U. Abbasi et al., Phys. Rev. Lett. 104, 161101 (2010).

[4] A. Aab et al., Physical Review D 90, no.12, 122005 (2014).

[5] P. Privitera et al., Internal Symposium on Future Directions in UHECR Physics (2012).

[6] D. Mandat et al., Journal of Instrumentation 12, T07001 (2017)

[7] M. Casolino, Proceedings of the 35th International Cosmic Ray Conference, 370 (2017)

[8] P. Klimov et al. Proceedings of the 35th International Cosmic Ray Conference, 1098 (2017)

[9] A.V. Olinto et al., Proceedings of the 35th International Cosmic Ray Conference, 542 (2017)

[10] Y. Tameda, et al., Progress of Theoretical and Experimental Physics, 2019, Issue 4, $043 F 01$ (2019).

[11] H. Tokuno, et al. , Nucl. Instr. Meth. A 676, 54 (2012).

[12] Y. Tameda, et al., Nucl. Instr. Meth. A 609, 227 (2009).

[13] T. Tomida et al. , Nucl. Instr. Meth. A 654, 653 (2011).

[14] A. Okumura et al., Astroparticle Physics 76, 38-47 (2016).

[15] D. Heck et al., Forschungszentrum Karlsruhe Report FZKA 6019 (1998). 